\title{
Density-Driven Segregation in Binary and Ternary Granular Systems ${ }^{\dagger}$
}

\author{
Kit Windows-Yule* and David Parker \\ ${ }^{1}$ School of Physics and Astronomy, University of Birmingham, UK
}

\begin{abstract}
We present a first experimental study of density-induced segregation within a three-dimensional, vibrofluidised, ternary granular system. Using Positron Emission Particle Tracking (PEPT), we study the steady-state particle distributions achieved by binary and ternary granular beds under a variety of differing system parameters. In doing so, we determine the extent to which the segregative processes in ternary systems resemble their comparatively well-researched binary counterparts. We examine the influence of particle elasticity on the system, demonstrating the existence of a significant parameter range for which the effects of inelasticity-induced segregation may be safely neglected. Finally, we investigate the existence of a causal link between convective motion within a system and the segregative behaviours observed.
\end{abstract}

Keywords: granular, segregation, binary, ternary, convection, buoyancy

\section{Introduction}

Granular materials, systems comprising multiple discrete, macroscopic components or 'grains', display a multitude of interesting physical phenomena both similar to and distinct from those observed in conventional 'molecular' materials (Jaeger et al., 1996). Of these myriad phenomena, perhaps the most pertinent to real-world applications (Muzzio et al., 2002; Mohabuth et al., 2007) is that of granular segregation, the process by which an energised granular system composed of two or more distinct particle 'species' may spontaneously separate into its individual constituents. Having no analogy in classical, molecular materials, this partitioning process of granular segregation remains incompletely understood. This lack of understanding is due, in part, due to the significant number of distinct mechanisms by which segregation can occur: particles may separate from one another due to differences in size (Rosato et al., 1987), density (Hong et al., 2001), elasticity (Serero et al., 2006; Brito and Soto, 2009) or shape ( $\mathrm{Li}$ et al., 2013) to name but a few. Nonetheless, thanks to the considerable volume of research on the matter, a reasonable level of comprehension of the segregative processes within simple, bidisperse systems has been achieved. However, in many real-world applica-

\footnotetext{
Received 2 April 2014; Accepted 3 June 2014

J-STAGE Advance Published online 30 June 2014

Edgbaston, B15 2TT, UK

* Corresponding author: Kit Windows-Yule;

E-mail: windowsyule@gmail.com

TEL:+44-0-414-4708
}

tions, particulate systems possess more than two distinct particle types. This poses an important question-when the condition of bidispersity is relaxed, is our knowledge based on these simple, two-component systems still relevant? In this paper, we take an incremental yet important step towards answering this question by comparing results obtained from an experimental, ternary-by-density granular system to new and existing data concerning similar, binary systems. In our experiments, the energy necessary to produce segregation is provided by vertical vibration of the experimental granulate. Vertical vibration is widely used in industrial processes requiring the separation and mixing of powders, grains and other particulate materials. Such vibrations can also occur inadvertently, for example during the transport of granular materials, leading in many cases to unwanted separation or mixing behaviours. Despite the clear importance of a better understanding of vibrated granular mixtures, there exist very few prior studies of these systems in the ternary case with, to the best of the authors' knowledge, no existing literature regarding the rôle of differing particle densities within these systems.

In this paper, we begin by describing the experimental system from which our results are acquired (Section 2) and detailing the manner in which data is extracted from this system (Section 3). We then provide information regarding the discrete particle simulations used to support and verify our findings (Section 4) before presenting and interpreting our results for both non-convective (Section 5.1) and convective (Section 5.2) binary and ternary systems. Finally, in Section 6, we summarise the major 
esiltstand conclusions arising from this work.

we demonstrate that while non-convective systems exhibiting spatially homogeneous packing densities and temperatures ${ }^{1)}$ undergo a relatively simple segregative process reminiscent of Archimedean buoyancy in classical fluids, spatially non-uniform and convective systems display significantly more complex segregation phenomena. We show that, in both cases, these segregative behaviours remain qualitatively similar in both two- and threecomponent systems, providing support for the possibility that our understanding of binary systems may, with further research, be successfully extended to the ternary case.

\section{Experimental Details}

Our experimental set-up consists of a granular bed of $N=60003 \mathrm{~mm}$ diameter, spherical particles housed within a cuboidal container which is vibrated sinusoidally in the vertical $(z)$ direction. Two main types of bed are investigated: binary, in which $N_{A}=N_{B}=3000$, and ternary, in which $N_{A}=N_{B}=N_{C}=2000$, where $A, B$ and $C$ denote individual particle species. In our experiments three species of particle, each of equal size but differing in their material properties, are used: steel $\left(\rho=7900 \mathrm{kgm}^{-3}\right)$, glass $\left(\rho=2500 \mathrm{kgm}^{-3}\right)$ and polyurethane $\left(\rho=1200 \mathrm{kgm}^{-3}\right)$. For brevity and ease of comprehension, the steel, glass, and polyurethane particles are referred to throughout the paper as heavy (H), medium (M) and light (L) particles, respectively.

In both the binary and ternary case, the total number of particles, $N$, and hence the dimensionless resting bed height, $H$, are held constant. The consistency in total bed height between binary and ternary systems is important, as $H$ is a key control parameter in determining the state of the system (Luding et al., 1994); the fixed H-value for the various systems explored thus allows a more direct comparison between the two cases. Similarly, the key control parameters relating to the mechanical excitation of the system (Eshuis et al., 2005), namely the frequency, $\omega=2 \pi f$, and peak amplitude, $A_{0}$, of the vibrations with which the system is driven, are also held equal for binary and ternary systems for all data sets.

In order to produce and observe a variety of behaviours within the experimental system, the dimensionless accel-

\footnotetext{
1) The temperature to which we refer here, and throughout this manuscript, is the granular temperature (Ogawa, 1978), defined as $T=m\left\langle c^{2}\right\rangle$, where $c$ is the fluctuation velocity of a particle about its mean value. The 'ordinary' or thermodynamic temperature can safely be assumed negligible when considering the behaviour of granular systems due to the large sizes, and hence high energies, of the particles involved (Jaeger et al., 1996). The granular temperature is an important quantity to consider, as it affects many aspects of a granular system's behaviour, including segregative processes (Lim, 2010).
}

eration, $\Gamma=\frac{\omega^{2} A}{g}$, is varied in the range $\Gamma \in(5,17)$ to produce varying degrees of fluidisation. Two differing container widths $L_{x}=L_{y}=40 \mathrm{~mm}$ and $L_{x}=L_{y}=80 \mathrm{~mm}$ are also utilised, providing bed heights $H=33.8$ and $H=8.4$ for the narrower and wider systems respectively. The variation of the container width not only varies the resting bed height, altering once again the density state of the system, but also determines the importance of wall effects, allowing us to investigate both convective and non-convective systems (see Fig. 1). For both system widths, $L_{z}=200 \mathrm{~mm}$. The considerable height of the container results in a minimal probability of particle collisions with the upper boundary, allowing the system to be considered effectively open. The relatively large size of the particles allows us to neglect air effects within the system (Zeilstra et al., 2008). The size of the particles combined with the use of a steel-based container will also act to reduce the influence of electrostatic effects within the system (Shi et al., 2007). The absence of significant effects due to interstitial air and the build-up of static charge is supported by the strong agreement observed between experiment and simulation, in which no interstitial air or electrostatic effects are included.

In this study, our main interest is in the equilibrium distribution of particles, or in other words the steady-state degree of segregation exhibited by the granular bed. For each data set, the system is initially vibrated for a period of $1000 \mathrm{~s}$ to ensure that a steady state is reached. The duration of this initial excitation was chosen based on
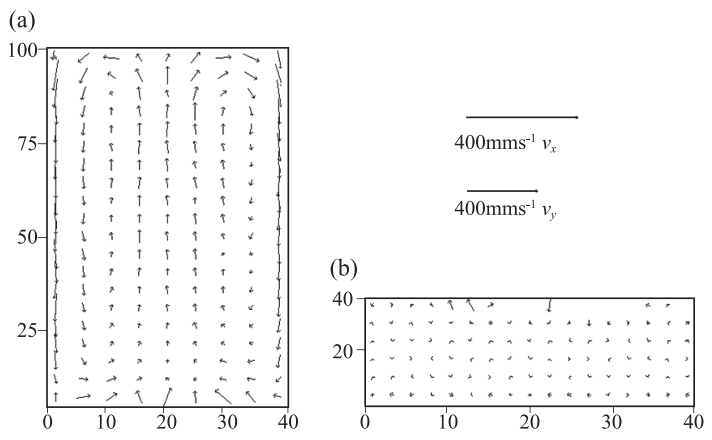

Fig. 1 Comparison of experimentally acquired velocity fields for a) convective and b) non-convective ternary systems of widths $L_{x}=L_{y}=40 \mathrm{~mm}$ and $L_{x}=L_{y}=80 \mathrm{~mm}$ respectively, each driven with a dimensionless acceleration $\Gamma=17$. The lengths and orientations of the arrows in the vector plots represent, respectively, the magnitude, $|v|$, and direction of the average velocity for a particle travelling through a given spatial region of the system. The images are scaled such that $|v|$ is directly comparable for the two systems shown. Note the considerably reduced magnitudes and random orientations of the velocity vectors in image b), indicative of the chaotic motion of particles within this system and its spatial homogeneity. 
pudiminary tests in which ternary beds of height $H=33.8$ and $H=8.4$ were driven with a constant acceleration $\Gamma=5$ over a period of $3600 \mathrm{~s}$. By utilising both the weakest driving and most polydisperse systems used in experiment, we can safely assume that these 'test runs' will provide an upper limit for the time required to reach a steady state (Windows-Yule et al., 2014). The point at which the system may be considered to be in a steady state is determined by subdividing the data set acquired into a series of $200 \mathrm{~s}$ segments and, for each of these segments, analysing the distributions of particle density and temperature. If the form of these distributions is found to remain consistent over multiple adjacent segments, then the system can be assumed to have reached a steady state. Over several repeated tests, both systems were typically found to achieve a non-equilibrium steady state in less than $200 \mathrm{~s}$, which was then maintained for the remainder of the $3600 \mathrm{~s}$ run. However, in order to thoroughly ensure reliability, an initial excitation period of a considerably longer duration was used when obtaining the main data sets. For all data sets presented, the steady-state nature of the system was confirmed as described above.

Preliminary tests were also conducted to investigate the influence of a system's initial particle distribution on the final steady state reached. Various systems - both convective and non-convective, and using both the highest and lowest excitation strengths explored in experiment-were investigated. In each case, three different preparation protocols were used: in the first protocol, the granular bed is stirred to produce a random distribution of particles, i.e. a mixed bed. In the second, a pure layer of heavy $(\mathrm{H})$ particles rests atop a pure layer of light (L) particles, and in the third a layer of $\mathrm{L}$ particles lies above a layer of $\mathrm{H}$ particles. Although, clearly, the time required for each system to reach its equilibrium distribution was affected by the system's initial conditions, the resultant steady state was not found to be affected in any of the tested cases. For the main experiments, each system was prepared using the first protocol.

The definitions of all symbols used in the manuscript may be found in Table 1 below.

\section{Data Acquisition-Positron Emission Particle Tracking}

Information is acquired from the experimental system using the Positron Emission Particle Tracking (PEPT) technique. This technique uses a modified dual-headed gamma camera to track, in three dimensions, the motion of a single particle which has been radioactively 'labelled' with a positron emitting radioisotope. By placing a granular bed containing such a radioactive 'tracer particle' between the twin detectors of the gamma camera and
Table 1 Definitions of symbols used

\begin{tabular}{|c|c|c|}
\hline Symbol & Definition & Units \\
\hline$A_{0}$ & Amplitude of base vibration & $\mathrm{m}$ \\
\hline$d$ & Particle diameter & $\mathrm{m}$ \\
\hline$H$ & $\begin{array}{l}\text { Dimensionless resting bed height- - height } \\
\text { of the static bed normalized by the particle } \\
\text { diameter }\end{array}$ & - \\
\hline$I_{\mathrm{s}}$ & $\begin{array}{l}\text { Segregation intensity, as defined in Section } \\
\text { 5.1. }\end{array}$ & - \\
\hline$f$ & Frequency of base vibration & $\mathrm{Hz}$ \\
\hline$L_{i}$ & $\begin{array}{l}\text { Dimensions of system in the } i \text {-direction of } \\
\text { the Cartesian coordinate system }(i=x, y, z)\end{array}$ & $\mathrm{m}$ \\
\hline$N$ & Total particle number & - \\
\hline$N_{i}$ & Number of particles of a given species $i$ & - \\
\hline$T$ & $\begin{array}{l}\text { Granular temperature, as defined in } \\
\text { Section } 1 .\end{array}$ & $\mathrm{J}$ \\
\hline$\Gamma$ & $\begin{array}{l}\text { Dimensionless acceleration of base } \\
\text { vibration }\end{array}$ & - \\
\hline$\varepsilon$ & Effective elasticity & - \\
\hline$\varepsilon_{i}$ & $\begin{array}{l}\text { Effective elasticity for a collision between } \\
\text { like particles of species } i\end{array}$ & - \\
\hline$\varepsilon_{i j}$ & $\begin{array}{l}\text { Effective elasticity for a collision between } \\
\text { unlike particles of species } i \text { and } j\end{array}$ & - \\
\hline$\mu$ & Coefficient of friction & - \\
\hline$\rho$ & Material density & $\mathrm{kgm}^{-3}$ \\
\hline$\phi$ & $\begin{array}{l}\text { Fractional concentration of a given particle } \\
\text { species in a multi-component system }\end{array}$ & - \\
\hline
\end{tabular}

determining the points at which the back-to-back $511 \mathrm{keV}$ $\gamma$-rays emitted by the tracer are detected, it is possible to triangulate the spatial position of this particle to an accuracy of up to $1 \mathrm{~mm}$ (Parker et al., 2002). For an adequately active tracer, several thousand of these triangulation events may be recorded each second, allowing the motion of the particle to be tracked with a temporal resolution of the order of milliseconds. Since the $\gamma$-rays used are highly penetrating, particle motion may be accurately recorded deep within the interior of even large, dense and/or opaque systems. A simple visual representation of the process described above may be seen in Fig. 2.

For systems in a non-equilibrium steady state (NESS), such as those discussed here, the long-time averaged motion of a single particle may, through the principle of ergodicity, be considered representative of the behaviour of the system as a whole (Wildman et al., 2000). For example, the average fraction of time spent by a single particle in a given region of the system (i.e. the tracer's residence time in this region normalized by the total duration of the data set) is directly proportional to the local 
For full details pertaining to the PEPT technique and its application to composite systems, the reader may refer to the works of Parker et al. (2002), Wildman et al. (2000) and Wildman and Parker (2002).

\section{Data Acquisition-Simulations}

Additional information and verification of experimental results is provided through the use of the MercuryDPM simulation code developed by the University of Twente (University of Twente, 2014; Weinhart et al., 2012; Thornton et al., 2012, Krijgsman et al., to be published). Although the bulk of the discussion presented in this manuscript pertains to experimentally acquired results, the ancillary inclusion of discrete particle method simulations allows certain parameters (e.g. coefficients of restitution), which are highly difficult to isolate in an experimental situation, to be individually varied and hence their effects studied. The high level of control provided by these simulations therefore allows us to test and verify hypotheses based on our experimental results. Simulations were conducted using experimental values of $f, A, N, d, H, L_{x}, L_{y}, L_{z}, \rho$ and $\varepsilon$. For collisions between particles of differing species, an inter-species coefficient of restitution was defined as $\varepsilon_{A B}=\left(\varepsilon_{A}+\varepsilon_{B}\right) / 2$, where $A$ and $B$ represent individual particle species. This definition of $\varepsilon_{A B}$ as the geometric average of the particles' individual elasticity values arises naturally from the spring-dashpot model of particle restitution (Cundall, 1979; Luding, 2008). For all instances in which simulated data is presented or discussed, unless specifically stated otherwise, the value of the frictional coefficient, $\mu$, may be assumed equal to 0.1 (Louge, 1999). Simulations were, however, conducted for a variety of $\mu$ values $\mu \in(0,1.0)$ in order to assess the impact of this parameter on the system's behaviour; this variation in the bed's frictional properties was found to have no significant impact on the system's segregative behaviour, simply acting as an additional source of dissipation, analogous to an increase in $\varepsilon$.

For full details of the MercuryDPM code, please refer to Thornton et al. (2013).

\section{Results and Analysis}

\subsection{Non-Convective Systems}

In a vibrofluidised granular bed where convection and other instabilities are absent, one can consider the system's temperature and packing density to be approximately uniform in the horizontal direction. This situation greatly simplifies the behavior within such a system. As such, we shall analyse first our observations for systems 
where rive the driving conditions are such that these instabisities are frustrated, before moving on to discuss the more complex, convective case.

Previous studies of bidisperse systems have shown that for systems whose temperature and density may also be taken as homogeneous in the vertical direction, segregative processes within granular fluids are governed simply by Archimedean buoyancy, much like their molecular counterparts (Huerta et al., 2005). In other words, a particle within a system whose conditions match those described above will experience an upward force proportional to the mass of material displaced by said particle ${ }^{3)}$. Thus, within such systems, one may expect particles lighter and/or larger than the average to rise towards the upper regions of the system and vice-versa. However, due to the non-uniformity with which they are driven and the dissipative nature of the particles of which they are composed, granular systems excited by vertical vibration often display density- and temperature-gradients in the $z$-direction (Helal et al., 1997), removing the condition of vertical homogeneity. Due in part to the presence of energy non-equipartition between the different components of a granular mixture (Feitosa and Menon, 2002), these gradients may significantly affect the segregation of a system (Luding et al., 2000; Garzó, 2006). Through the careful choice of system parameters, we create vibrofluidised granulates which display near-homogeneous temperature fields in both horizontal and vertical directions, as well as systems displaying more prominent vertical temperature gradients. Thus, we can explore each of the segregative mechanisms described above, and hence determine whether they might be applicable also to ternary systems. Fig. 3 provides visual representations of the temperature distributions discussed above. The experimental temperature profiles illustrated in Fig. 3 are created, as with the density profiles discussed previously, through a subdivision of the experimental volume into multiple, equally-sized cells. For all data points falling within a given cell, the instantaneous particle velocity is determined, and thus the mean velocity within this particular cell calculated by averaging over the number of data points recorded. The deviations of the individual velocities from this average value can then be used to compute the local temperature, and the process repeated for each of the system's cells, allowing the spatial variation of the granular temperature to be visualised. Fig. 3(a) shows a typical case for which one may assume a constant temperature, i.e. vertical temperature homogeneity. Although the profile displays a non-trivial gradient at large $z$, com-

\footnotetext{
3) In granular systems, of course, one must account not only for the material density of the particles themselves but also the solids fraction of the system, which will both influence the 'effective density' of the displaced material.
}

(a)
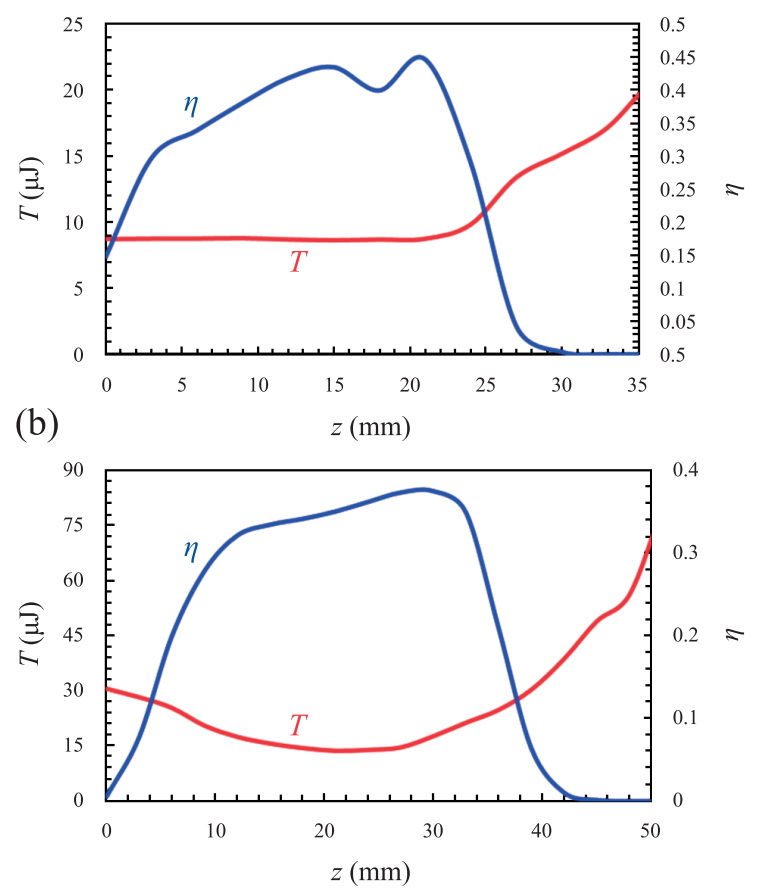

Fig. 3 Experimental vertical packing fraction, $\eta$, (blue) and temperature, $T$, (red) profiles for ternary systems excited with dimensionless accelerations of a) $\Gamma=11$ and b) $\Gamma=17$.

parison with the system's packing profile clearly demonstrates the fact that only a small fraction of particles exist in this region of temperature non-uniformity. Fig. 3(b), meanwhile, shows an example of a system in which temperature gradients may be expected play a significant role. Here, it can be seen that $T$-gradients at both large and small $z$ extend far into the bulk of the system.

Plots showing the variation of the fractional concentrations, $\phi$, of individual particle species for different regions within the experimental system can be seen in Figs. 4-6. Due to the symmetry of the system, the data shown here in the $x-z$ plane is identical to that observed in the $y-z$ plane. Comparison of the binary system shown in Fig. 6(a) to the equivalent ternary system presented in Fig. 4 demonstrates a qualitatively similar behavior in both cases: the lightest particles predominantly occupy the higher regions of the bed, while the heaviest species typically sink to the bottom of the system. In other words, our results (both those shown in the Figures provided as well those which, for brevity, are not explicitly presented) strongly imply that ternary systems, like binary systems, exhibit Archimedean buoyancy in the absence of strong temperature gradients. Similarly, the behavior observed in Fig. 6(b) shows a qualitative similarity to that demonstrated in Fig. 5. This is a highly pleasing - and potentially important - result, as the observed similarities in behavior between bi- and tri-disperse systems demon- 


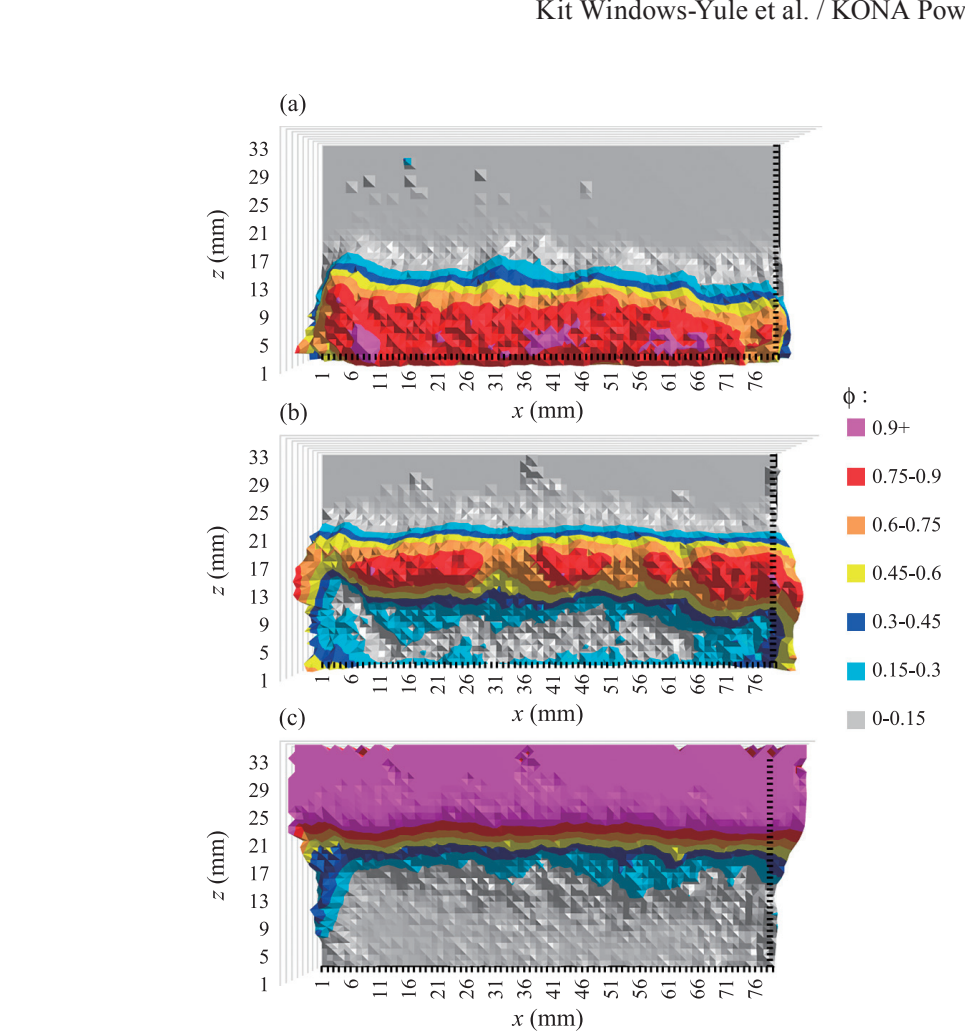

Fig. 4 Two-dimensional particle concentration distributions for an experimental ternary system. Data is shown for a) steel $(\mathrm{H})$ particles, b) glass (M) particles and c) polyurethane (L) particles in a binary system with $H=8.4$ particle diameters and $L_{x}=L_{y}=80 \mathrm{~mm}$. The system is driven with a dimensionless acceleration $\Gamma=11$.

strates the possibility that theories developed for simple two-component systems may be relatively easily extended to ternary and, potentially, even higher-order systems. However, considerable further research is required in order to substantiate this postulation. The findings described above are also found to hold in simulation for arbitrary combinations of particle density; although segregation is less complete for cases in which differences between particles' densities are less pronounced, the lightest component will invariably possess the highest vertical centre of mass and the heaviest the lowest.

Of course, the extension of our understanding of binary systems to the ternary or polydisperse case is still by no means trivial. Nonetheless, data for the relatively uncomplicated case of purely buoyancy-driven segregation seemingly demonstrate a remarkably straightforward correspondence between the segregative behaviors exhibited by a ternary system and those of the equivalent binary systems comprising the same components. An example of this can be seen in Fig. 7, which shows the vertical distributions of individual particle species for a ternary (LMH) system of steel $(\mathrm{H})$, glass $(\mathrm{M})$ and polyurethane (L) particles alongside the corresponding binary steel/glass (MH) and glass/polyurethane (LM) systems. In order to allow a quantitative comparison between the binary and ternary

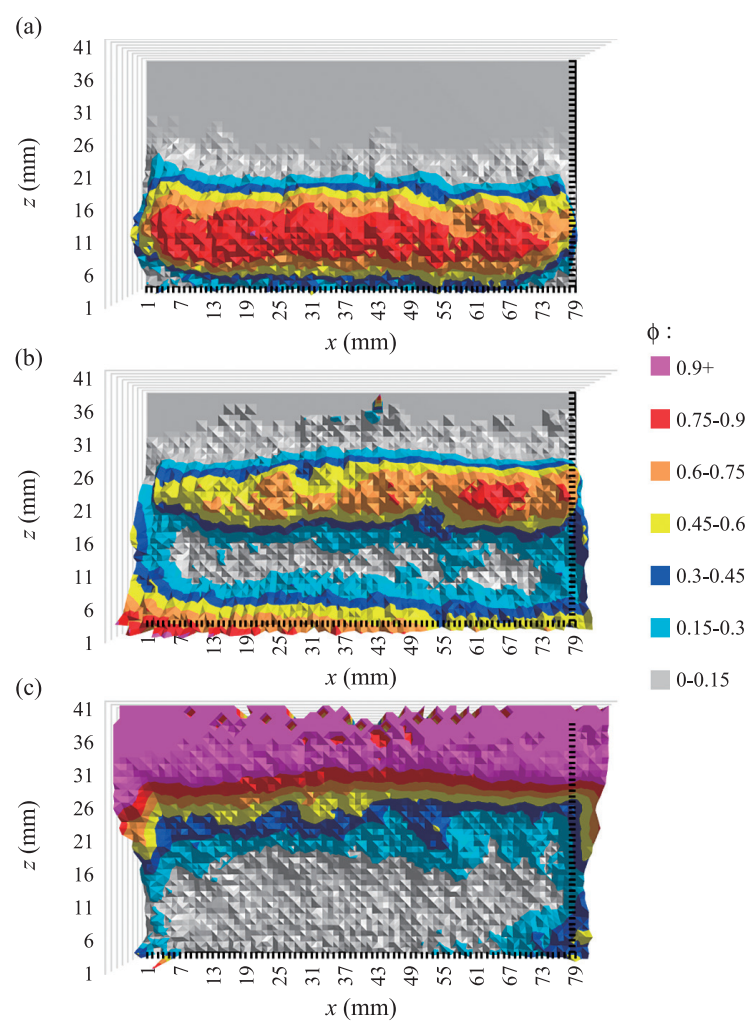

Fig. 5 Two-dimensional particle concentration distributions for a) steel $(\mathrm{H})$ particles, b) glass $(\mathrm{M})$ particles and c) polyurethane (L) particles in an experimental ternary system driven at $\Gamma=17$. All other parameters are identical to those given in Fig. 4.

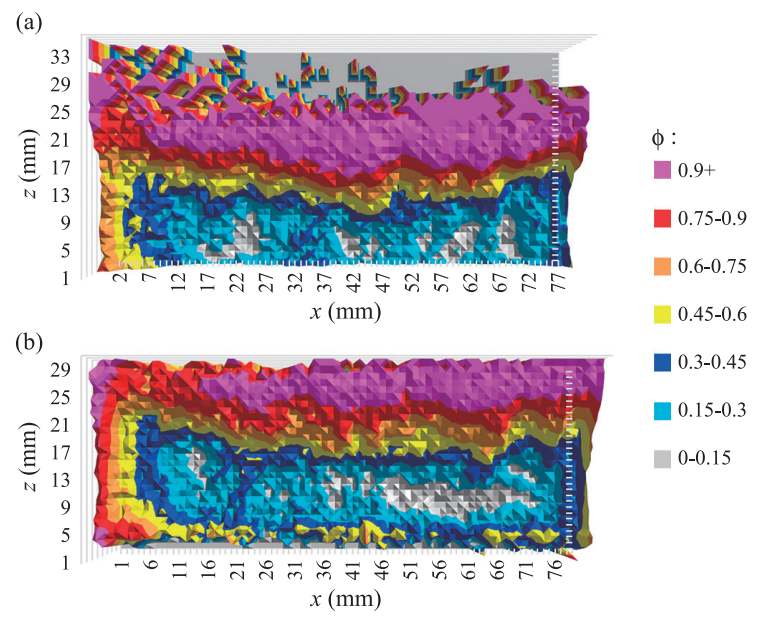

Fig. 6 Two-dimensional particle concentration distributions for the lighter component of a binary glass/steel mixture, corresponding to experimental data. Results are shown for the cases a) $\Gamma=11$ and b) $\Gamma=17$.

cases, we define a quantity $I_{\mathrm{S}}$, the segregation intensity, which serves as a measure of the degree to which a single component of a system becomes segregated from the other species (Khakhar et al., 1997). $I_{\mathrm{S}}$ is measured by dividing the experimental volume into a series of $N_{C}$ 
(b)

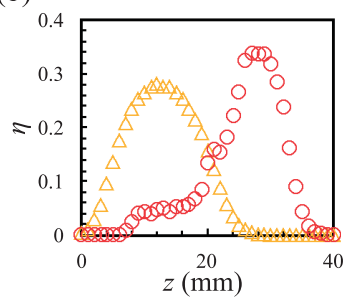

(c)

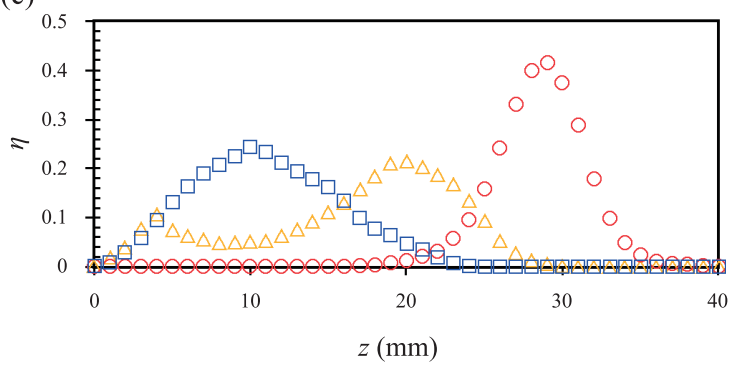

Fig. 7 Experimentally acquired one-dimensional vertical packing fraction profiles showing the distribution of individual particle species for a) a binary system of steel (blue squares) and glass (orange triangles) particles, b) a binary system of glass (orange triangles) and polyurethane (red circles) particles and c) a ternary system consisting of steel, glass and polyurethane particles. In all cases, the dimensionless acceleration $\Gamma=14$.

equally sized 'cells' and, for each cell, calculating the local concentration, $\phi_{i}$, of an individual particle species, which can then be compared to the mean concentration, $\phi_{m}$, of the system as a whole. Specifically, the segregation intensity is defined as:

$$
I_{S}=\left[\frac{\sum_{i=1}^{i=N_{C}}\left(\phi_{i}-\phi_{m}\right)^{2}}{N_{C}}\right]^{1 / 2}
$$

The specific maximal value of $I_{\mathrm{S}}$ (achieved when a system's components are entirely separated from one another) is dependent on the number of species within the system, and the relative volume fraction of each species. Therefore, for all data presented, the calculated segregation intensity is normalized by the appropriate maximal value such that a value $I_{\mathrm{S}}=1$ corresponds to a completely segregated system, while $I_{\mathrm{S}}=0$ denotes the case of perfect mixing. For the binary systems shown in Figs. 7(a) and 7 (b), we find that $I_{\mathrm{S}} \approx 0.62$ for the $\mathrm{H}$ component of the $\mathrm{MH}$ system, and $I_{\mathrm{S}} \approx 0.82$ for the L component of the LM system. For the ternary system, meanwhile, we find $I_{\mathrm{S}} \approx 0.62$ for the $\mathrm{H}$ component and $I_{\mathrm{S}} \approx 0.86$ for the L component. In order to test whether this close correspondence between $I_{\mathrm{S}}$ values for binary and ternary systems is a robust physical phenomenon or merely coincidence, a series of simulations were conducted using various values of particle density. The resultant data was found to strongly suggest that if the particle densities for all three species are adequately disparate, the values of segregation intensity for the $\mathrm{L}$ and $\mathrm{H}$ particles in the ternary system are approximately equal to the $I_{\mathrm{S}}$ values of their respective binary counterparts. However, if the density ratio between heavy and intermediate ( $\mathrm{H}$ and $\mathrm{M}$ ) or intermediate and light ( $\mathrm{M}$ and $\mathrm{L}$ ) particles is small enough that significant mixing occurs between the two species, the resultant segregation of the ternary system is found to differ. It should be noted that the precise density difference for which a correspondence in the $I_{\mathrm{S}}$ values of binary and ternary systems may be expected to vary dependent on the strength with which the system is driven and its resting height. Nonetheless, for the range of parameters investigated here it is found, from our simulations, that a density ratio above $\frac{\rho_{H}}{\rho_{L}} \sim 2$ is adequate to produce the above-described situation. However, in certain experimental systems, differences in particle elasticity may also be expected to play an important role in the system's mixing behaviours, and hence significantly alter this 'threshold value'. Once again, this is a topic worthy of further research.

In order to explain these findings, we consider two hypothetical systems: in system 1 , the mass of an L particle is considerably smaller than that of an $\mathrm{M}$ particle which, in turn, is considerably smaller than that of an $\mathrm{H}$ particle; in system 2, meanwhile, the mass of an L particle is considerably smaller than that of an M particle, while the mass differential between $\mathrm{M}$ and $\mathrm{H}$ particles is somewhat reduced. In system 1 , due to the large mass differences, mixing between any two species will be limited, resulting in a ternary system with relatively pure interfaces between $\mathrm{H}$ and $\mathrm{M}$ particles and between $\mathrm{M}$ and $\mathrm{L}$ particles. Thus, the buoyancy force acting upwards on the $\mathrm{L}$ particles in this three-component system will be simply equal to a certain displaced volume of pure $\mathrm{M}$ particles. Hence, the forces acting on the $\mathrm{L}$ particles in such a ternary system are identical to those in the binary ML system, resulting in a similar degree of segregation. In system 2, however, the smaller disparity in mass between $\mathrm{M}$ and $\mathrm{H}$ particles will result in a higher degree of mixing, thus removing the condition of pure boundaries between species. In this case, a mixture of $\mathrm{M}$ and $\mathrm{H}$ particles will be displaced by the L particles in the ternary system, resulting in an increased buoyancy force acting on the L component of the bed, and hence a correspondingly increased $I_{\mathrm{S}}$ compared to the equivalent binary LM case, as is observed in simulations. Through a similar process, for the case in which the mass difference between the $\mathrm{M}$ and $\mathrm{L}$ species becomes small enough to allow significant mixing in the upper region of the bed, one observes a decreased $I_{S}$ for the $\mathrm{H}$ component of a ternary system when compared to the equivalent binary $\mathrm{MH}$ system. In order to test this theory, simulations were per- 
or ilitis

formedfor a variety of combinations of particle density, with comparison being drawn between ternary beds corresponding to the situation of 'system 2' as described above and similar binary beds for which the density of the L component is held constant, while the density of a secondary, 'MH', species is set equal to the average of the $\mathrm{M}$ and $\mathrm{H}$ components. In these binary beds, the particle number ratio $N_{\mathrm{L}}: N_{\mathrm{MH}}$ was held equal to $1: 2$ to approximate the ternary situation. The observed similarities between the two systems provides qualitative support for our hypothesis.

It is hoped that, through further work stemming from this finding, it may be possible to construct a generalized theoretical framework capable of predicting, for the case in which there exists no gradient of granular temperature in the direction of gravity, the behavior of a ternary granular system based only on the knowledge of the segregative behaviors of the relevant binary systems.

Another interesting feature of Fig. 7 is the considerably increased average density for the $\mathrm{L}$ particles compared to the other two species. This observation is particularly surprising as one would expect the particles on the free surface of a system to be more easily able to expand and thus, if anything, exhibit a reduced density. The simple explanation for this observation is that the $\mathrm{L}$ particles used in experiment are considerably less elastic than the other species used. This lower elasticity leads to an increased degree of dissipation, i.e. a greater loss of energy during intra-species collisions. This energy loss will lead to a smaller average separation between these inelastic particles, and hence the augmented packing fraction observed (Hopkins and Louge, 1991).

Since differences in particle elasticity have, both in simulation and experiment, been shown to significantly influence the segregative behaviours of granulates (Serero et al., 2006; Brito and Soto, 2009; Windows-Yule et al., 2014), simulations were conducted using a variety of particle elasticities, $\varepsilon$, in order to assess the role of dissipation on the various systems under investigation. It is interesting to note that, for the range of parameters explored in this study, the elasticity of particles has no significant impact on the degree of segregation observed, despite altering the specific form of the particle distributions observed, as exemplified in Fig. 8. It is thought that this lack of influence due to elasticity effects is due simply to the large disparities in material density between particles and hence the dominance of segregation mechanisms due to mass differences.

\subsection{Convective Systems}

We now consider systems exhibiting the commonly observed convective instabilities caused by frictional interactions between particles and the boundaries by
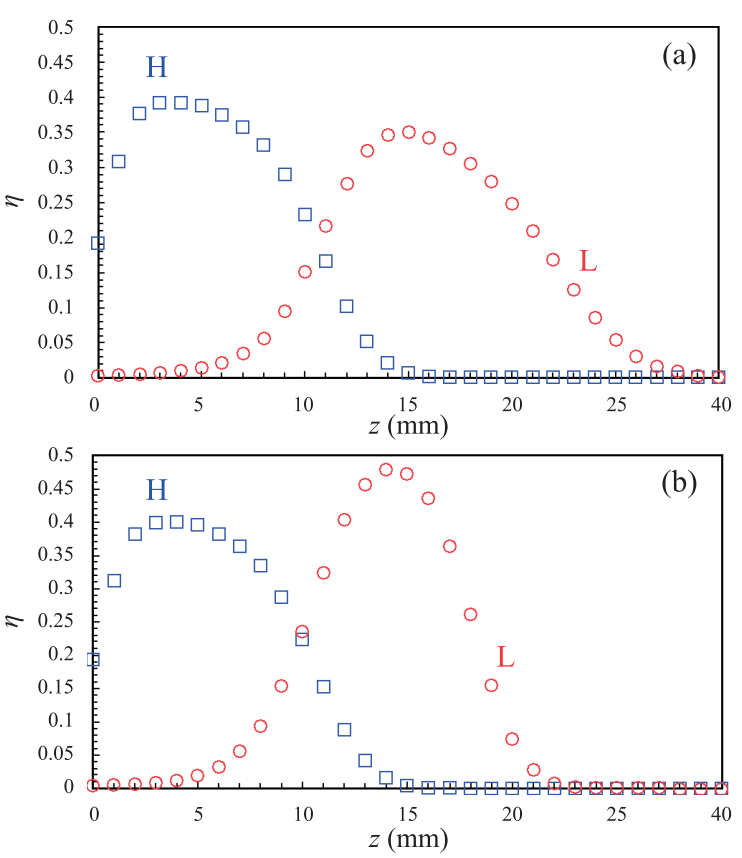

Fig. 8 Simulated density profiles for a binary system of polyurethane (L, red circles) and steel ( $\mathrm{H}$, blue squares) particles vibrated with dimensionless acceleration $\Gamma=8$. The systems in images a) and b) are identical other than the elastic coefficient, $\varepsilon$, of the L component of the system, which is implemented as $\varepsilon=0.9$ in case a) and $\varepsilon=0.3$ in case b). Despite this considerable difference in $\varepsilon$, and the resultant differences in the forms of the profiles, the values of segregation intensity, $I_{\mathrm{S}}$, remain strikingly similar in both cases-specifically, $I_{\mathrm{S}}=0.83$ in case a) and 0.84 in case b). This effective invariance in $I_{S}$ with $\varepsilon$ is observed over a range of driving accelerations $\Gamma \in(8,17)$ and particle elasticities $\varepsilon \in(0.1,0.9)$.

which they are constrained (Brown, 1939; Knight, 1997). In order to investigate the effect of convective motion on the distribution of particles within bi- and tri-disperse beds, we now study a narrower, taller system wherein wall effects are considerably more prevalent, rendering the system more susceptible to convective instabilities. As is immediately obvious from Figs. 9 and 10, the equilibrium distribution of particles achieved by the convective system significantly differs from the case in which the system is approximately spatially homogeneous in terms of its granular temperature and packing density. At first, the particle distribution seems considerably more complex and difficult to explain than that of the previously discussed non-convective case; for instance in the systems shown in Fig. 10, the vertical centre of mass position of the heavy species is in fact slightly higher than that of the light or intermediate particles, in seeming defiance of the principle of minimum energy. However, when one compares the particle distributions of Figs. $\mathbf{9}$ and $\mathbf{1 0}$ to the velocity fields presented in Fig. 11 and the granular temperature profile shown in Fig. 12, the mechanisms underlying these particular configurations become clearer. 
(a)

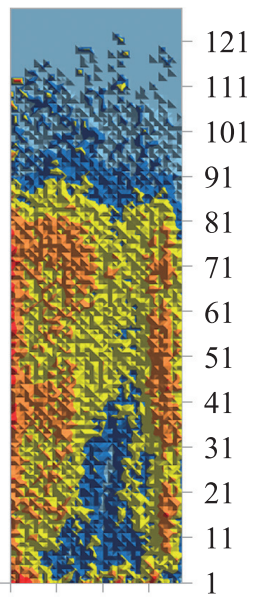

1112131

$x(\mathrm{~mm})$ (b)

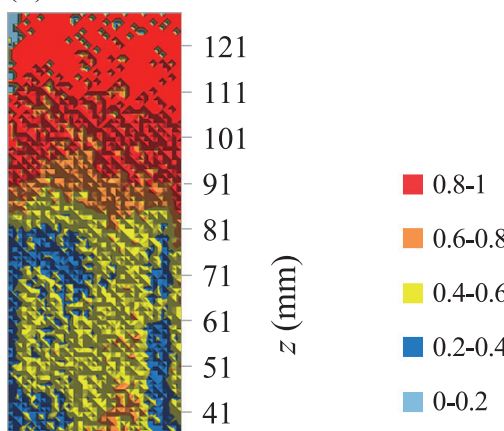

Fig. 9 Two-dimensional particle concentration distributions for an experimental binary system showing the fractional concentrations of a) steel $(\mathrm{H})$ particles, b) polyurethane (L) particles. Here, the resting bed height $H=33.8$ and the container width $L_{x}=L_{y}=40 \mathrm{~mm}$. The system is driven with a dimensionless acceleration $\Gamma=11$

Instantly notable from Figs. 9(a) and 10(a) is the tendency of the $\mathrm{H}$ particles to largely occupy the regions corresponding to the centres of convection rolls. The light particles, meanwhile, are generally excluded from these areas, occupying instead the relatively fast-flowing outer regions of the convective streams in the centre and at the edges of the experimental system. This behavior is observed for all convective systems investigated, across the entire range of driving accelerations used. In all cases, an energetic 'gas' comprising almost entirely L particles may be observed in the uppermost section of the bed; a large proportion of lighter ( $\mathrm{L}$ and $\mathrm{M})$ particles is also found near the base of the system, where the proximity to the vibrating base creates a region of relatively high energy (see Fig. 12). In the ternary systems, the M particles, as one might predict, show somewhat of a composite behavior; in the bulk of the bed, the M particles occupy the intermediary regions of the convective stream, where the convection centres are largely dominated by the heavier particles, and the fastest-flowing regions mainly comprise L particles. In the energetic upper regions of the bed, where particle motion more closely resembles that of a disordered fluid, we also find $\mathrm{M}$ particles to be present. In this region, the system's behavior is more reminiscent of that described in section 5.1, with the lightest particles simply tending to rise to the top, and the heaviest sinking. It should be noted that the general behaviours noted here were consistently observed over a range of driving parameters, specifically $8 \leq \Gamma \leq 17$. It is also worth noting that all possible binary combinations of particles (i.e. glass/steel, polyurethane/steel, polyurethane/glass) were tested; for each pairing, the resultant granulate was found to behave in a manner qualitatively similar to that described above irrespective of the specific density combinations used, providing evidence for the generality of our observations.

Although similar behaviour has been observed previously in constrained, bidisperse beds (Yang, 2006; Tai et al., 2010), the current study provides several new insights into this segregation phenomenon: firstly, and perhaps most importantly to the aims of this work, we show that the migration of heavy particles towards a system's convection centres occurs in ternary as well as binary systems. Secondly, we demonstrate that the observations made previously in quasi-two-dimensional systems apply also in fully three-dimensional systems such as those described here. We also show the behavior to be applicable for a variety of material densities and elasticities and, indeed, combinations thereof. Another important finding of the current study is the strong evidence of cause and effect in granular systems exhibiting both convection and segregation: although we have so far demonstrated the coexistence of segregation and convective motion in our system, and a correspondence between the two, we are yet to provide specific evidence as to a causal link between these phenomena. For instance, is the particle distribution within the system produced by the convective flow present, as one might intuitively expect? Alternatively, is it possible that an initial segregation of heavier particles may produce a localised region of higher density and lower temperature, as has been observed in previous studies (Rivas et al., 2011a,b), causing an instability within the system which subsequently leads to the 
(a)

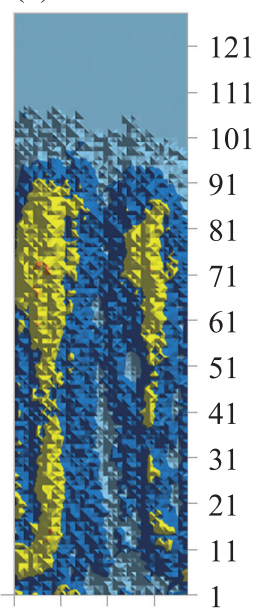

1112131 (b)

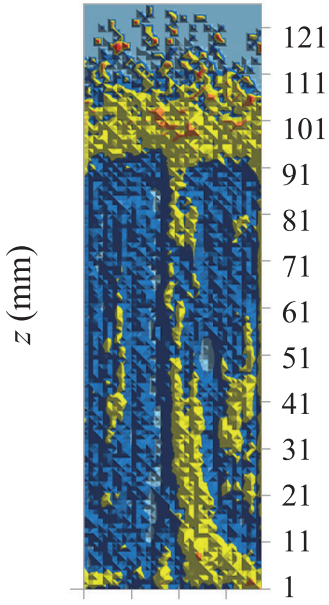

1112131 (c)

$\begin{array}{llll}1 & 11 & 2131\end{array}$

$x(\mathrm{~mm})$

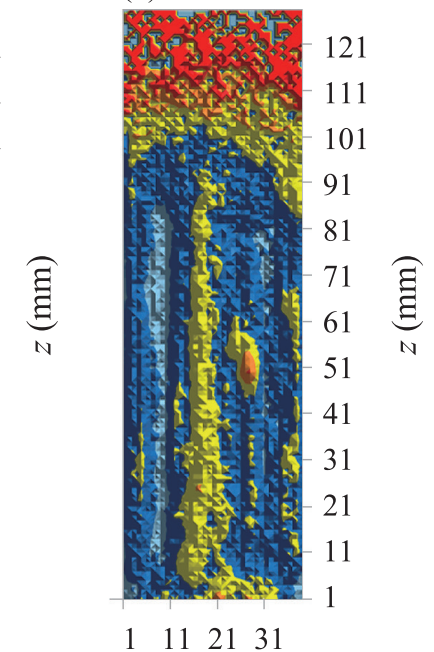

$x(\mathrm{~mm})$

$x(\mathrm{~mm})$

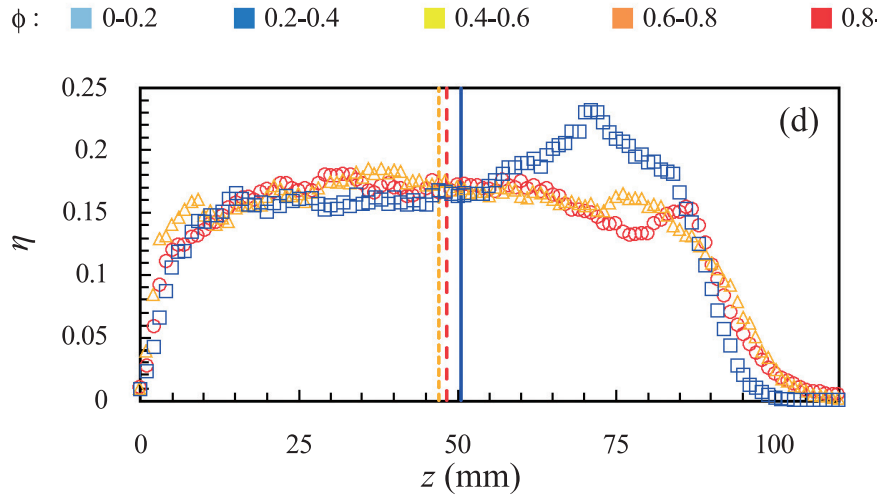

Fig. 10 Panels a)-c): Experimental results showing two-dimensional particle concentration distributions for a) steel (H) particles, b) glass (M) particles and c) polyurethane (L) particles in a ternary system with $\Gamma=11$. All other parameters are identical to those given in Fig. 8. Panel d) shows the corresponding one-dimensional packing profiles, with $\mathrm{H}$ particles represented by blue squares, $\mathrm{M}$ by yellow triangles and $\mathrm{L}$ by red circles. The centres of mass of the H, M and L species are indicated by solid, dotted and dashed vertical lines respectively.

observed convective motion? Fig. 11 shows velocity vector fields for equivalent unary, binary and ternary systems. From these plots, the orientations of the velocity vectors make clear the presence of convection in each of the three cases. For all degrees of polydispersity, the major features of the convective flow - the height of the rolls, their orientation, the horizontal and vertical positions of the convection centres, the width of the central flow region etc.- - remain remarkably similar. This consistency in spite of the markedly different compositions of the systems in question strongly implies convection to be the cause and segregation the effect, rather than the convective motion observed being somehow a result of the particle separation. In other words, the fact that convection remains even in a monodisperse system, where segregation is, by definition, impossible, clearly demonstrates that this convective flow cannot be driven by, or indeed instigated by, segregative behaviours. Although this finding may not be particularly surprising, it is nonetheless important, as it provides substantial support for the idea of convection as a major mechanism in segregative processes.

Knowing that it is convection that causes segregation, and not the inverse, we now attempt to address the issue of how convective motion affects the distribution of particles within the system. Since a full theoretical treatment is beyond the scope of this current paper, we propose instead a simple, qualitative explanation. We posit that the distribution of particles within a convective granular bed is determined by the tendency of a physical system towards its lowest energy state, where mechanical stability may be achieved. Thus it seems reasonable that, in a given binary or ternary system, the heaviest particles migrate towards the slowest-moving regions, corresponding here to the areas at the centres of the convection rolls (see Fig. 11); 
(b)

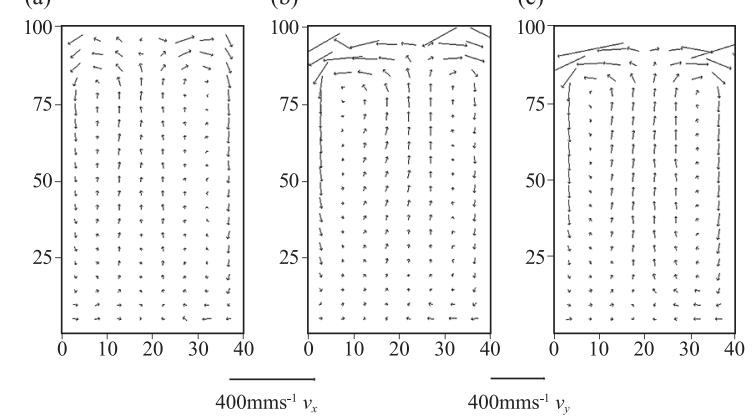

Fig. 11 Experimentally acquired velocity vector fields demonstrating the presence of convective motion in a) ternary, b) binary and c) unary systems. In all cases, the systems are driven with an acceleration $\Gamma=11$.

this localization of the $\mathrm{H}$ particles to low-velocity regions clearly results in a reduction of the system's average kinetic energy compared to, for instance, a homogeneous distribution of particles. Similarly, the light particles minimize kinetic energy through their domination of the dilute, high-velocity upper region of the system (see Fig. 12). It is interesting to note the parallel between our observations and those of Luding et al. (2000), who found that in granulates which were polydisperse by size, the larger-and hence, by definition, more massive-particles demonstrated a similar tendency to segregate into the 'colder' or lower-energy regions of a system.

An alternate view may be provided using the work of Rivas et al. (2011a, b) as a basis. Rivas et al. describe a clustering process whereby an increased pressure exerted by the more highly excited L particles will act to 'push' heavier particles away from regions of stronger agitation, thereby creating dense clusters of $\mathrm{H}$ particles. Since, for the systems described in the current section, the areas of lowest agitation correspond to the convection centres, the $\mathrm{H}$-clusters naturally form in these regions.

Further work, both experimental and simulational, is needed to verify which, if either, of the above-described mechanisms accurately describes and explains the observed behavior.

Whatever the underlying cause, a knowledge that the heaviest particles display a tendency to occupy the lowestvelocity regions and, conversely, the lightest particles the highest-velocity areas allows the prediction, at least in a qualitative manner, of the likely arrangement of differently massive particles in systems displaying higher degrees of polydispersity-i.e. the $2^{\text {nd }}$ heaviest species is likely to populate the $2^{\text {nd }}$ slowest region, the $3^{\text {rd }}$ heaviest the third slowest etc., until the positions of all particle species have been accounted for. The knowledge that such a correlation between local velocity and particle concentration exists, and the implication of its generality to ternary and higher-order granulates, may prove highly

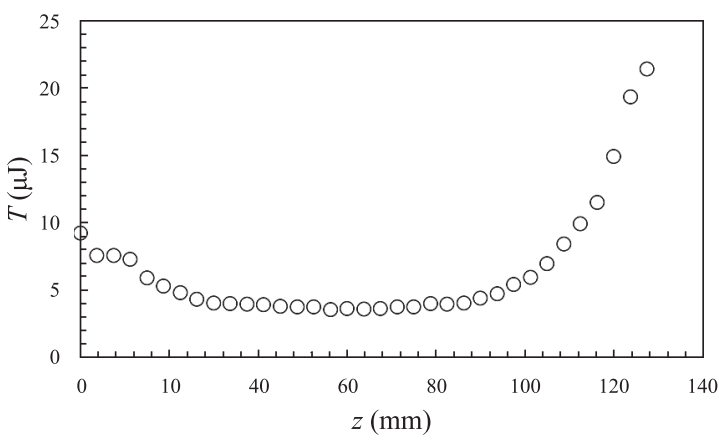

Fig. 12 Experimental vertical granular temperature profile for a ternary system driven with an acceleration $\Gamma=14$. The form of the profile is typical for the convective systems investigated here-a relatively high temperature for small $z$, i.e. near the energizing bottom plate of the container, decreasing to a roughly constant value throughout the main bulk of the system before increasing sharply at large heights where the particles form a dilute granular gas.

useful in the future construction of theoretical frameworks for polydisperse granular systems.

\section{Summary and Conclusions}

Through the experimental observation of a variety of monodisperse, binary and ternary granular systems, and the application of discrete particle simulations, we have studied the segregative behaviors of monosized granular beds in both convective and non-convective states.

We have provided a clear indication that a transition from a binary to a ternary system does not inherently change the mechanisms underlying the segregation of distinct particle species; this is an important result, as it suggests that existing findings relating to the segregation of bidisperse systems may be relatively simply extended to provide explanations and predictions of the behaviours of the comparatively less-researched ternary systems. The similarities in segregative behavior between bi- and tridisperse systems have been found to persist over a range of driving parameters, varying bed heights and aspect ratios and for systems both in the presence of and absence of convective motion.

We have demonstrated that, for the range of parameters explored here, segregative effects due to differences in particle elasticity are negligible; this direct demonstration of the ability to avoid inelasticity-induced segregation through careful choice of system parameters is potentially of use both in research and in industry.

Finally, for systems in which both convection and segregation are present, we have addressed the open question of cause and effect, demonstrating - through comparison of convective unary, binary and ternary systems - that it 
isdeon

is eonkective motion which leads to segregation, and not the inverse.

\section{Acknowledgements}

The authors would like to thank Nicolás Rivas for his highly valuable input. The authors would also like to gratefully acknowledge the financial support of the Hawkesworth scholarship, provided by the late Dr. Michael Hawkesworth. Finally, we thank Prof. Stefan Luding and the University of Twente for granting access to the computer facilities on which the simulations in this paper were performed.

\section{References}

Brito R., Soto R., Competition of Brazil nut effect, buoyancy, and inelasticity induced segregation in a granular mixture, Eur. Phys. J. Spec. Top., 179 (2009) 207-219.

Brown R.L., The fundamental principles of segregation. J. Inst. Fuel 13 (1939) 15-19.

Cundall P.A., Strack O.D.L., A discrete numerical model for granular assemblies, Geotechnique, 29 (1979) 47-65.

Eshuis P., van der Weele K., van der Meer D., Lohse D., Granular leidenfrost effect: Experiment and theory of floating particle clusters, Phys. Rev. Lett., 95 (2005) 258001.

Feitosa K., Menon N., Breakdown of energy equipartition in a 2D binary vibrated granular gas, Phys. Rev. Lett., 88 (2002) 198301.

Helal K., Biben T., Hansen J.P., Local fluctuations in a fluidized granular medium, Physica A, 240 (1997) 361-373.

Hong D.C., Quinn P.V., Luding S., Reverse Brazil nut problem: competition between percolation and condensation, Phys. Rev. Lett., 86 (2001) 3423.

Hopkins M.A., Louge M.Y., Inelastic microstructure in rapid granular flows of smooth disks, Phys. Fluids A, 3 (1991) 47-57.

Huerta D.A., Sosa V., Vargas M.C., Ruiz-Suárez J.C., Archimedes' principle in fluidized granular systems, Phys. Rev. E, 72 (2005) 031307.

Jaeger H.M., Nagel S.R., Behringer R.P., Granular solids, liquids, and gases, Phys. Today, 49(4) (1996) 1259-1273.

Khakhar D.V, McCarthy J.J., Shinbrot T., Ottino, J.M. Transverse flow and mixing of granular materials in a rotating cylinder, Phys. Fluids, 9 (1997) 31-43.

Knight J.B., External boundaries and internal shear bands in granular convection, Phys. Rev. E 55, (1997) 6016

Li C., Zhou Z., Zou R., Pinson D., Yu A., Discrete modelling of the packing of ellipsoidal particles, AIP Conf. Proc., 1542 (2013) 357-360.

Lim E.W.C., Density segregation in vibrated granular beds with bumpy surfaces, AIChE J., 56 (2010) 2588-2597.

Louge M.Y., in: http://www.mae.cornell.edu/microgravity/ impact-table.html, Cornell University, Ithaca, NY (1999).
Luding S., Herrmann H.J., Blumen A., Simulations of twodimensional arrays of beads under external vibrations: Scaling behaviour, Phys. Rev., E50 (1994) 3100.

Luding S., Strauss O., McNamara S., Segregation of polydisperse granular media in the presence of a temperature gradient, in: Rosato A.D. and Blackmore D.L. (Eds.), IUTAM Symposium on Segregation in Granular Flows, Dordrecht, Kluwer Academic Press, 2000, pp. 297-304.

Luding S., Cohesive, frictional powders: contact models for tension, Granular Matter, 10 (2008) 235-246.

Mohabuth, N., Hall, P., Miles N., Investigating the use of vertical vibration to recover metal from electrical and electronic waste, J. of Minerals Engineering, 20 (2007) 926-932.

Muzzio F.J., Shinbrot T., Glasser B.J., Powder technology in the pharmaceutical industry: the need to catch up fast, Powder Technol., 124 (2002) 1-7.

Ogawa S., Multitemperature theory of granular materials, Proceedings of US-Japan Seminar on Continuum-Mechanical and Statistical Approaches in the Mechanics of Granular Materials, Tokyo, 1978.

Parker D.J., Forster R.N., Fowles P., Takhar P.S., Positron emission particle tracking using the new Birmingham positron camera, Nucl. Instrum. Methods Phys. Res., Sect. A 477 (2002) 540-545.

Rivas N., Ponce S., Gallet B., Risso D., Soto R., Cordero P., Mujica N., Sudden chain energy transfer events in vibrated granular media, Phys. Rev. Lett., 106 (2011) 088001.

Rivas N., Risso D., Soto R., Cordero P., Energy bursts in vibrated shallow granular systems, AIP Conf. Proc., 1332 (2011) 184-189.

Rosato A.D., Strandburg K.J., Prinz F., and Swendsen R.H., Why the Brazil nuts are on top: Size segregation of particulate matter by shaking, Phys. Rev. Lett. 58 (1987) 1038.

Serero D., Goldhirsch I., Noskowicz S.H., Tan M.-L., Hydrodynamics of granular gases and granular gas mixtures, J. Fluid Mech. 554 (2006) 237-258.

Shi Q, Sun G., Hou M., Lu K., Density-driven segregation in vertically vibrated binary granular mixtures, Phys. Rev. E 75 (2007) 061302

Tai C.H., Hsiau S.S., Kruelle C.A., Density segregation in a vertically vibrated granular bed, Powder Technol., 204, (2010) 255-262.

Thornton A.R., Weinhart T., Luding S., Bokhove O., Modeling of particle size segregation: Calibration using the discrete particle method, Mod. Phys. C, 23 (2012) 1240014.

Thornton A.R., Krijgsman D., te Voortwis A., Ogarko V., Luding S., Fransen R., Gonzalez S.I., Bokhove O., Imole O., Weinhart T., A review of recent work on the Discrete Particle Method at the University of Twente: An introduction to the open- source package MercuryDPM, Discrete Element Methods 6, Conference Proceedings, 2013.

University of Twente, 2014, MercuryDPM homepage $<$ MercuryDPM.org>

Weinhart T., Thornton A.R., Luding S., Bokhove O., From discrete particles to continuum fields near a boundary, Gran. Mat. 14:2 (2012) 289-294.

Wildman R.D., Huntley J.M., Hansen J.-P., Parker D.J., Allen D.A., Single-particle motion in three-dimensional vibroflu- 
idized granular beds, Phys. Rev. E, 62 (2000) 3826.

W Wirefman R.D., Parker D.J., Coexistence of two granular temperatures in binary vibrofluidized beds, Phys. Rev. Lett., 88 (2002) 064301.

Windows-Yule C.R.K., Weinhart T., Parker D.J., Thornton A.R., Effects of Packing Density on the Segregative Behaviors of Granular Systems, Phys. Rev. Lett., 112 (2014) 098001.
Yang S.C., Density effect on mixing and segregation processes in a vibrated binary granular mixture, Powder Technol., 164 (2006) 65-74.

Zeilstra C., van der Hoef M.A., Kuipers J.A.M., Simulation study of air-induced segregation of equal-sized bronze and glass particles, Phys.Rev. E, 77 (2008) 031309.

\section{Author's short biography}
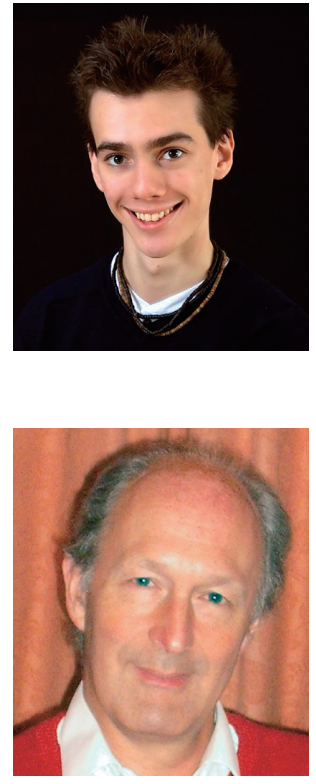

\section{Kit Windows-Yule}

Kit Windows-Yule is a doctoral researcher in the University of Birmingham's School of Physics and Astronomy. His current research concerns the characterisation of the various segregative processes exhibited by granular systems, with the eventual aim of understanding the fundamental mechanisms underlying these processes.

\section{David Parker}

David Parker graduated in Mathematics from Cambridge University and was then employed for 10 years as an experimental nuclear physicist at the UKAEA's Harwell Laboratory. During this period he was awarded his DPhil from Oxford University for studies of nuclear reaction mechanisms, and also worked on industrial applications of nuclear physics. Since 1989 he has been in the School of Physics and Astronomy at the University of Birmingham, where he is currently Professor of Physics and Director of the Positron Imaging Centre. He was responsible for developing the PEPT technique, and has applied it to study a wide range of granular and fluid flow phenomena. He also supervises the Birmingham MC40 Cyclotron, used for a variety of research and commercial work. 